\title{
Effect of the wave length on the Görtler instability
}

\author{
F. Bahri and Y. Kohama \\ Institute of Fluid Science, Tohoku University \\ Katahira 2-1-1, Sendai, Japan.
}

\begin{abstract}
The stability of boundary layer, in presence of curved streamlines, where longitudinal vortices are developed was studied experimentally. The centrifugal instability of boundary layer generated by Görtler mechanism represents one fundamental case to understand the breakdown process of streamwise vortices in the turbulence. Streamwise vortices were triggered by a wire grid fixed upstream of the leading edge with an imposed wave length. The smoke visualization reveals that new vortices appear at the mid-distance of the imposed wavelength if it accedes a critical value. The secondary instability of the vortices depends strongly by the nature of the boundary layer developed.
\end{abstract}

Ke y words: Boundary layer, streamwise vortex, Görtler vortices, wave length, secondary instability.

\section{Introduction}

Primary and secondary Görtler instabilities are studied in reviews by Hal1, Li u ${ }^{2}$, Floryan ${ }^{3}$ and Saric ${ }^{4}$. However, the secondary instability was documented theoretically by Sabry \& Liu ${ }^{5}$, Lee \& Liu 6 , Guo \& Finlay ${ }^{7}$, Bottaro \& Klingmann ${ }^{8}$, Benmalik \& Saric ${ }^{9}$, and $\mathrm{Li} \& \mathrm{Malik}^{10}$. All those computations predict the secondary instability and indicate that the highly inflectional profiles in a shear layer in both spanwise and wall-normal directions are the origin of that instability. Probably The completed computation was performed by $\mathrm{Yu} \& \mathrm{Liu}^{11}$. They included the effects of the viscosity and described the secondary instability. They also, performed an energy balance and concluded that the sinuous mode predominate the varicose one. The secondary instability has been also carried out in some experimental investigations and concluded with different observations. Bippes ${ }^{12}$ found that the amplification of Görtler vortices is in accord with the linear theory and the sinuous mode of the secondary instability manifests before the breakdown to the turbulence. Aihara et al ${ }^{13}$ discovered a 'horseshoe' type of the secondary instability. Ito ${ }^{14}$, Kohama ${ }^{15}$, Peerhossaini \& Wesfreid ${ }^{16}$ and Bahri et al $17-18$ measured the flow ficld of the 'mushroom-like' structures and observed the development of sinuous and varicose modes. Although, the secondary instability was largely documented in the work Swearingen and Blackwelder 22 . They observed both modes and they were able to detect the origin of that instability and identify the mechanism of each mode. They found that the sinuous mode was associated to the $\mathrm{dU} / \mathrm{dz}$ and the varicose mode was related to $\mathrm{dU} / \mathrm{dy}$. The work of Swearingen \& Blackwelder provided an important background for comparisons with theory and computations.

In order to reproduce the different modes of secondary instability, in this paper we performed many smoke visualizations with same conditions as experiment of Bahri $^{18}$ (radius of curvature, freestream velocity and wavelength). We specially focus on the dependence of the wavelength on the structure of the boundary layer and the influence of that wavelength on the secondary instability.

\section{Experimental apparatus}

The visualizations are performed in a new low-speed open-loop wind tunnel shown schematically in Figure 1. It consists of a $120 \mathrm{~cm}$ square cross-section settling chamber which contains honeycombs and screens. Downstream, the flow is accelerated through a threedimensional contraction of $18 / 1$ ration. The test section consists of a curved test section fabricated completely from Plexiglas to conduct several visualizations carefully. The radius of the curvature is $650 \mathrm{~mm}$. The test section has a streamwise length of $1700 \mathrm{~mm}$ and a cross-section of $150 \mathrm{~mm}$ by $380 \mathrm{~mm}$ width. The control of the streamwise distribution of the static-pressure was assured by holes of $0.7 \mathrm{~mm}$ diameter drilled on the centerline of the curved wall of the test section. Artificial trigging device to fix streamwise vortices are introduced in order to measure the dependence of that wavelength on the secondary instability stage. The spanwise wavelength was fixed to $30 \mathrm{~mm}$ and generated 
through a 0.2 -mm-diameter wire grid positioned upstream of the leading edge.

In this investigation, all visualizations are performed by injecting smoke through a carefully machined slot of $0.7 \mathrm{~mm}$ thickness inclined at $45^{\circ}$ and situated at $50 \mathrm{~mm}$ from the stagnation line in the leading edge. The freestream velocity is fixed at $2 \mathrm{~m} / \mathrm{s}$. The boundary layer is enlightened by an Argon laser sheet oriented normally to the wall at each expected streamwise location. Görtler vortices are viewed by a CCD camera mounted perpendicularly to the plane of the laser sheet.

\section{Results}

Recently, Li and Malik (1995) (as well as others) computed the nonlinear development of the fundamental secondary instability modes. They performed different calculations with wavelengths of $0.9,1.8$ and $3.6 \mathrm{~cm}$ as measured in the experiment of Swearingen and Blackwelder (1987). They observed that the region between mushrooms called 'valley' becomes much wider as the wavelength increases. They calculated the energy of different modes and they found, for the longerwavelength case $\lambda=1.8 \mathrm{~mm}$, that both mode zero (mean flow correction) and the second mode are important. They concluded that the secondary instability modes depend strongly upon the Görtler vortices' wavelength initially generated.

In order to study the dependence of the wavelength on the nonlinear regime as well as on the secondary instability an intensive program of flow visualization was carried out. Visualizations are reproduced in the same environment as reference ${ }^{18}$. That experiment reproduced 'mushroom-like' structures for the streamwise velocity due to the action of Görtler vortices. Hot-wire measurement of the streamwise velocity, in the cross-section, are presented in figures 2(a)-(d) by for different streamwise locations.

That measurements show, in figure 2-(c), a small growth of the boundary layer thickness at the middle of the wavelength imposed initially at $3 \mathrm{~cm}$. However, $\mathrm{Li}$ and Malik (as well as others) observed a uniform boundary layer thickness in the 'valley' region, figure 3 , for large-wavelength case $\lambda=3.6 \mathrm{~cm}$.

In order to access the secondary instability, we focus on that difference observed in the 'valley ' region and we plot the iso-contours of the velocity gradient $\partial \mathrm{U} / \mathrm{h}$ calculated from the data of figure 2-(c). Over the pairs of vortices, one can see, in figure 4 , that the iso-lines of $\partial U / \partial$ change the sign in the 'valley' region which indicate the existence of one inflexion point. That observation lets suggest that there is a new vortex that starts to give birth at the middle of the initial wavelength.

In this new investigation, the extended streamwise length of the test section of $1700 \mathrm{~mm}$ permits to fellow carefully the growth of the vortices. The visualization reveals that new vortices appear in the center of wavelength and confirm our hypothesis. Figures $5(a)$ (o) show the spatial development of three pairs of Görtler vortices in the streamwise direction.
The cross-section visualization of the boundary layer shows that the triggered vortices start formation from $\mathrm{x}=200 \mathrm{~mm}$ to $\mathrm{x}=350 \mathrm{~mm}$ presented in figures 5 . Fron $x=400 \mathrm{~mm}$, figure 5 , we observe small concentration of smoke in the valley region, which indicates that the velocity is not homogenous distributed over that region. That smoke concentration testify that a new 'pick', region of relative low streamwise velocity, will gives 'birth'. In figures 5, relevant to distances from $x=400$ to $600 \mathrm{~mm}$, we observe the growth of that new vortices.

Peerhossaini \& Bahri 20 show that at the earlier stage the vortices developed in the linear regime if the smoke visualization indicate a small concentration. However, if the 'mushroom-like' is formed, the spanwise profiles of mean streamwise component present is distorted and the flow enter in the nonlinear regime. Thus, in visualizations of figure 5 both observations are present in the distance from $x=400$ to $500 \mathrm{~mm}$. At that region, we presume that the growth of initial vortices (developed by the imposed wavelength $\lambda=30 \mathrm{~mm}$ ) is nonlinear. However, the new vortices (developed by the half of wavelength) still in the linear regime. From $x=500$ to $600 \mathrm{~mm}$, we observe the nonlinear growth of both vortices (new and old). we observe some oscillations of the secondary instability after $x=600 \mathrm{~mm}$ and we are not able to identify the exact mode of that secondary instability. Perhaps that is the consequence of the structure of the boundary layer scenario.

this suggestion makes difficult to observe complicate the oscillation of the secondary instability. After $x=600 \mathrm{~mm}$, in figures 5 , we observe that the flow inter in the transition to the turbulence.

\section{Conclusion}

In this investigation, the extended streamwise length of the test section permits to fellow the growth of the vortices. The visualization reveals that a new vortices appear in the valley region when the wavelength is large. Other revelation is the linear and nonlinear growth of vortices during the same streamwise distance. The oscillation and the mode of the secondary instability can not clearly identified due to the complexity of the structure of the boundary layer.

This phenomenon should gives a new approach of the disturbance amplification in the streamwise direction and modifies the classic growth and breakdown to the turbulence.

\section{References}

[1] Hall, P. (1988) "The nonlinear development of Görtler vortices in growing boundary layers", J. Fluid Mech. 193, 243-266.

[2] Liu, J. C. T (1991) "On Scalar Transport in Nonlinearly Developing Görtler Vortex Flow". Geophys. Astrophys. Fluid Dynamics, Vol. 58,pp.133-145

[3] Floryan M 1991 "On the Gortler instability of boundary layer" Prog. Aerospace Sci., 28. 235-271

[4] Saric W 1994 "Gortler Vortices" An. Rev, Fluid Mech,26. 
[4] Saric W 1994 "Gortler Vortices" An. Rev, Fluid Mech,26.

[5] Sabry, A. and Liu, J. C. T. (1991) "Longitudinal vorticity elements in boundary layers: nonlinear development from initial Görtler vortices as a prototype problem", J. Fluid Mech Vol.231, pp.615-663

[6] Lee \& J T C Liu 1992 "On the growth of mushroom like structures in nonlinear spatially developing Gortler vortices flow" Phys. Fluids A.Vol;4 \#1, pp 95-103

[7] Guo Y \& Finlay. W. H 1994, "Wavenumber selection and irregularity of spacially developing nonlinear Dean and Gortler vortices" J. F. M. 264,pp 140

[8] Bottaro, A (1994) "Streamwise Vortices in Fluid Flow: Instabilities and Breakdown" IMHEF Report T94-26 December 1994

[9] W. Saric \& A. Benmalek 1991 "A Gortler vortices with periodic curvature"Bound layer stability \& transition ASME Vol;114

[10] Li, F and M, Malik 1995 "Fundamental and subharmonic secondary instability of Gortler vortices." J. F. M. 296 pp 77-100

[11] Yu, X. and Liu, J. C. T. (1994) "On the mechanism of sinuous and varicose modes in threedimensional viscous secondary instability of nonlinear Görtler rolls", Phys. Fluids 6, 736.

[12] Bippes, H. \& H Gortler 1972 "Dreidimensionale strungen in der grenzschicht an einer konkaven wand", Acta Mech, 14

[13] Aihara \& Koyama 1981 "Secondary instability of Gortler vortices" Trans. Jap. Sco. Aero Space 24, 78-94 [14] Ito, A. (1985) "Breakdown structure of longitudinal vortices along a concave wall", J. Japan Soc. Aero. Space Sci. 33, 166-173.
[15] Kohama, Y (1987) "Three dimensional boundary layer transition on a concave-convex curved wall" Turbulence management and relaminarization, Proceeding of the IUTAM Symposium, H.W. Lipmann, R. Narasimba, Eds., Springer-Verlag, pp: 215-226

[16] Peerhossaini H. \& Wesfreid, J.1988 "On the inner structure of streamwise Gortler rolls", Int.J. Heat \& Fluid flow, 9, p 12.18,

[17] Bahri.F, Kestoras, Ajakl. and Peerhossaini, $\mathrm{H}$ (1994) "An experimental investigation of the growth of the Görtler vortices: the fluid mechanics study in view of the heat transfer study". EUROMECH 327, 25-27 August, Kiev (Ukraine),

[18] Bahri.F (1995) "Etude experimentale de l'instabilité de Görtler dans une couche limite concave: régime linéaire et non-linéaire", Ph.D. Thesis, Ecole Centrale de Nantes, Universite de Nantes, France.

[19] Bahri.F and Peerhossaini, H (1994) :Eigenfunctions of Görtler vortices in a highly non linear regime; an experimental approach". EUROTHERM 39, 12-13 December Nantes (France).

[20] Peerhossaini, H and Bahri.F (1995) "Experiments o n mode decomposition in nonlinear Görtler instability". Presented a $t$ : gth $^{\text {th }}$ Couette-Taylor Workshop August 7-10. , Boulder, Colorado, [21] Peerhossaini, H and Bahri.F (1995)."Experiments on Fourier expansion description of velocity in Görtler vortex flow". Presented at: $48^{\text {th }}$ Annual meeting, Division of Fluid Dynamics American Physical Society, November 19-21 Irvine, CA. USA

[22] Swearingen, J.D. and Blackwelder R. 1987., The growth and breakdown of of streamwise vortices in the presence of a wall, J. Fluid Mech. 182, pp 255-292.

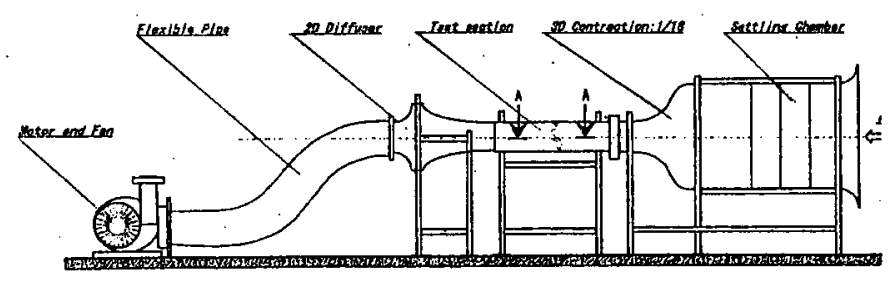

Figure 1: Schemalite of the low-speed open-loop wind lunnel
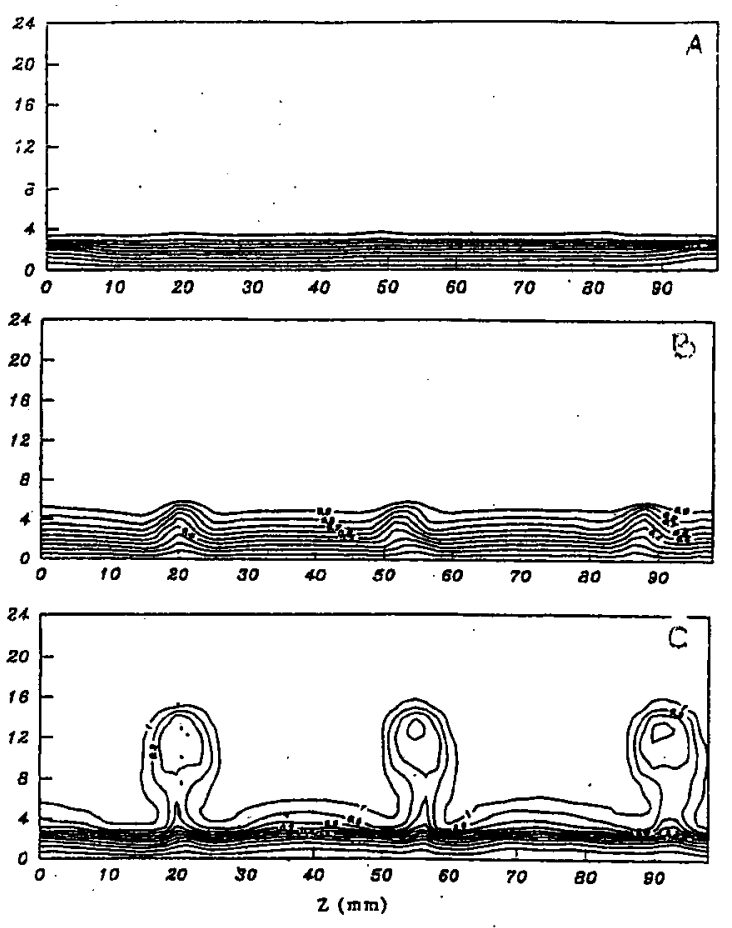

Iso-contours of mean streamwise velocity in the cross-section plene for different $x$-locations 

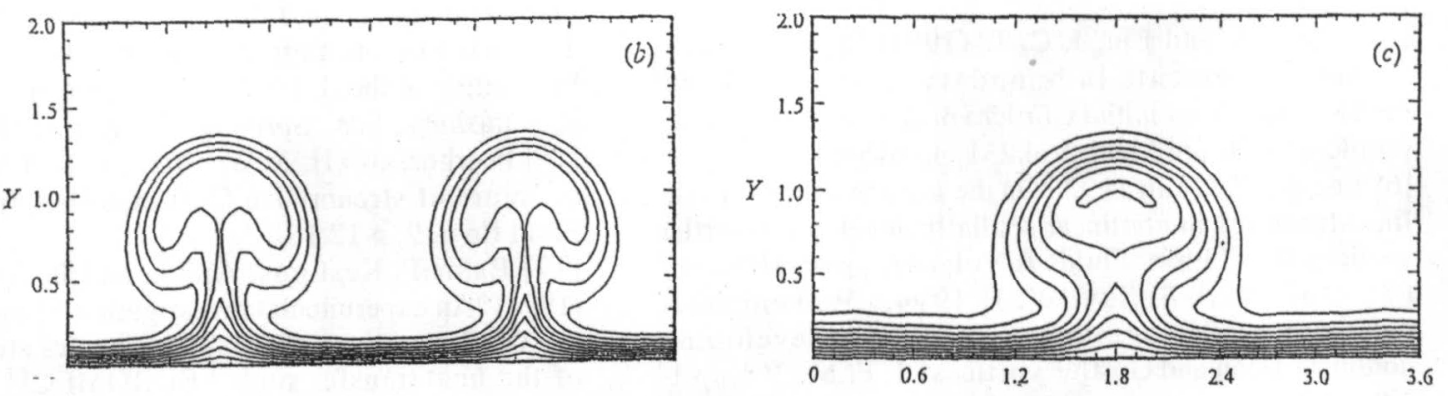

$\mathrm{fig}^{3}$ : Iso-contours of mean streamwise velocity in the cross-section plane for different wavelength.

(b): $\lambda=1.8 \mathrm{~cm}$ and $(\mathrm{c}): \lambda=3.6 \mathrm{~cm}$

From: ref. [10] Li \& Malik

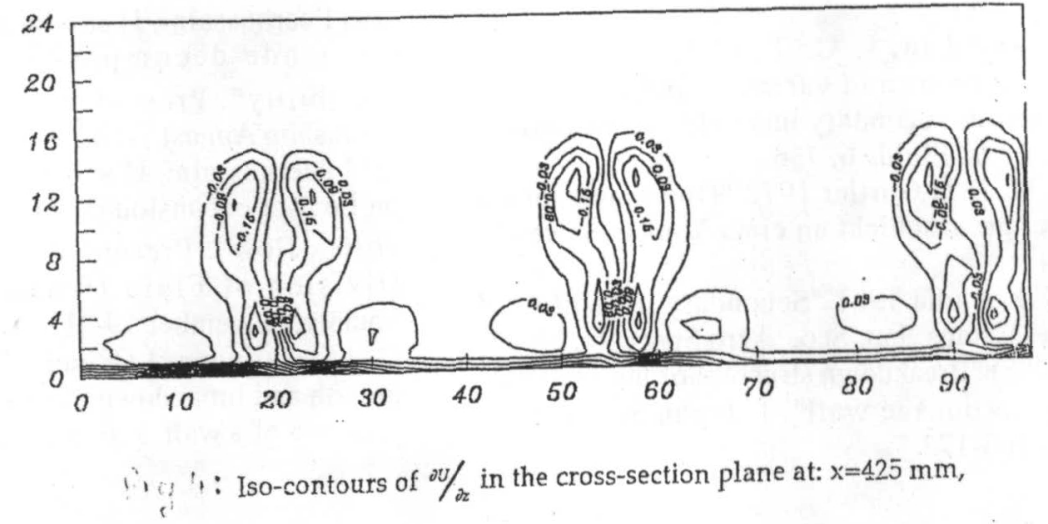

$\mathrm{x}=200 \mathrm{~mm}$

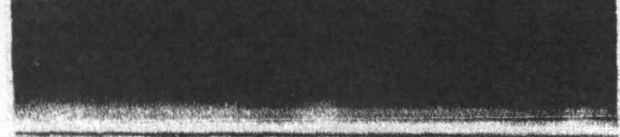

$\mathrm{x}=350 \mathrm{~mm}$

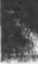

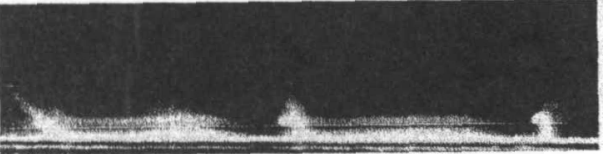

$\mathrm{x}=40() \mathrm{mm}$

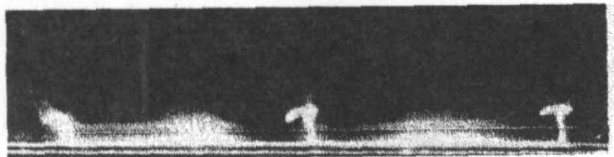

$\mathrm{x}=600 \mathrm{~mm}$

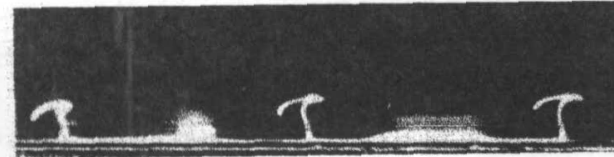

$x=500 \mathrm{~mm}$

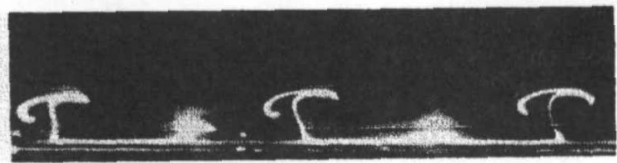

$\mathrm{x}=550 \mathrm{~mm}$
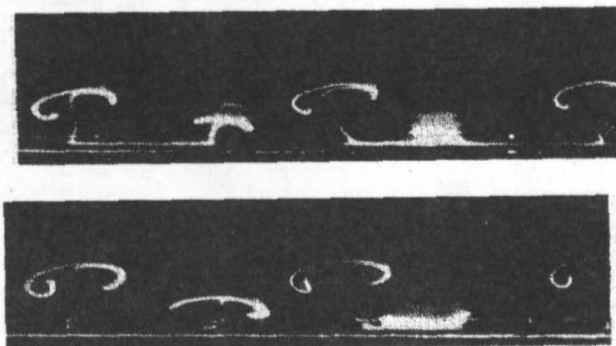

Figure 5: Spacial evolution of Gortler vortices. 